N. E. Piskunov, W. W. Weiss, D. F. Gray, eds.

\title{
Stellar Photospheres: Success, Failure, Ambiguity, \& Ambition
}

\author{
David F. Gray \\ Department of Physics $\mathcal{E}$ Astronomy University of Western Ontario
}

\begin{abstract}
.
Models are compared with observations in several examples, including spectroscopic temperature measurement, irradiance variations, stellar rotation from line profiles, and velocity fields in stellar photospheres. A few key issues for investigations in the near future are suggested.
\end{abstract}

\section{Introduction}

Sometimes we need to pause and review "life." Why do we study stellar atmospheres? If there are 150 participants here, we likely have about 150 answers to the question. Chemical composition of stellar atmospheres is enlisted for studies of nucleosynthesis, galactic structure, or the mechanics of convective envelopes. Polarization tells us about magnetic fields. Shapes of spectral lines tell us about atmospheric velocity fields, rotation rates, magnetic fields, and pressure broadening. Positions of spectral lines also tells us about velocity fields, and about companions in orbit. Bumps passing through line profiles are used to map stellar surface features.

Some of us just want to understand the nature and structure of stellar atmospheres and the physical processes in them: radiation transfer, velocity fields, heterogeneous structure, magnetic regeneration, chromospheric and coronal heating and structure, mass loss, angular momentum dissipation, formation of open field lines and interplanetary fields, and so on. Wow! There is no shortage of reasons.

Now I had originally organized this talk around the four categories listed in the title, but then I realized how dangerous that could be should some of you not like the category in which I placed your work! So, instead, let's look at a few selected topics.

\section{Spectroscopic Temperature Measurement}

Modeling the Balmer line wings has become a powerful way to measure temperatures of cool stars (Gehren 1981, Fuhrmann et al. 1994, van't Veer-Menneret et al. 1998, Gardiner et al. 1999). Recent improvements in the line-absorption coefficient (Stehlé 1994, Stehlé \& Hutcheon 1999, Barklem et al. 2000a, Barklem et al. 2000b, Barklem et al. 2002) give fairly reasonable absolute stellar temperatures (see Fig. 1), although the scale seems to be too low by $\sim 35 \mathrm{~K}$. Errors on individual stars are still moderately high, $\sim 100 \mathrm{~K}$, and there are inconsistencies 

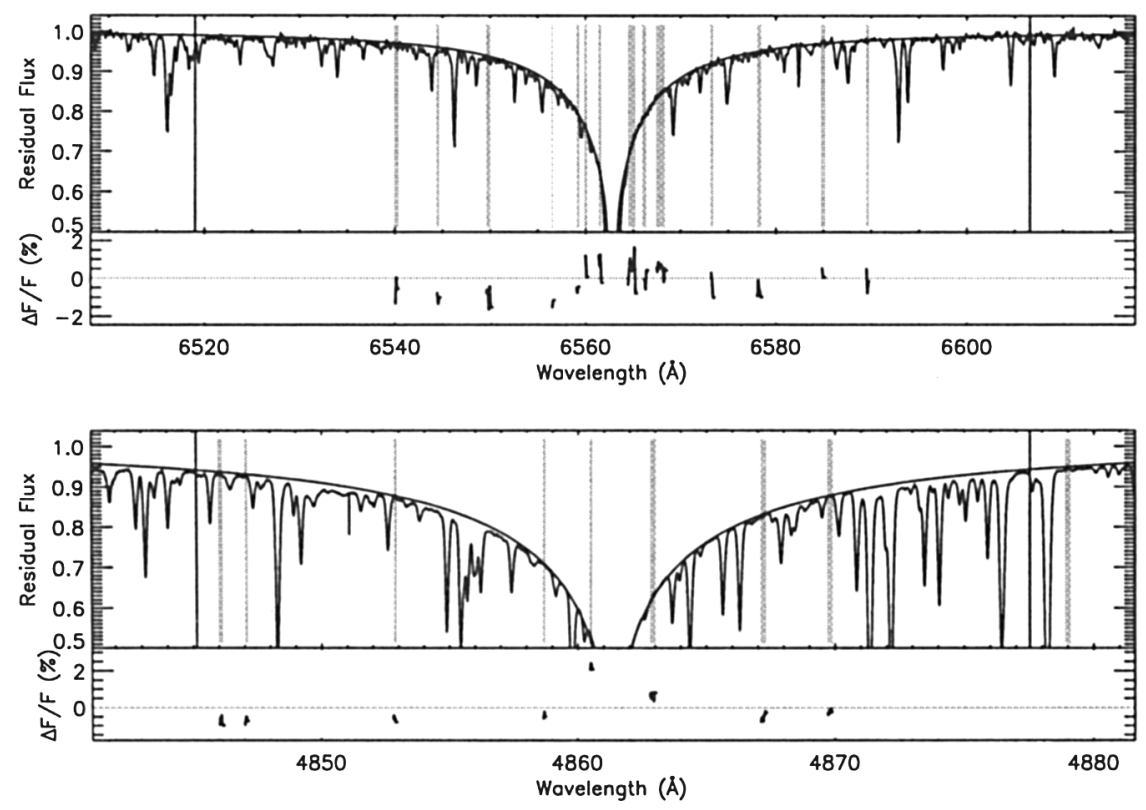

Figure 1. The $\mathrm{H} \alpha$ (top) and $\mathrm{H} \beta$ (bottom) profiles for Procyon (HR 2943, F5 IV-V) are well modeled to yield an effective temperature of $6520 \pm 80 \mathrm{~K}$ (from Barklem et al. 2002). Note the difference in wavelength scales for the two lines. Mismatches are shown in the lower $(\Delta F / F)$ panels.

with $\mathrm{H} \alpha$ giving $\sim 65 \mathrm{~K}$ higher values than $\mathrm{H} \beta$. Improved spectroscopy will help; it is a daunting task to measure such wide lines because of various field errors in spectroscopic measurements. Line blending is also a problem, leaving only small windows where the actual wings of the hydrogen lines can be seen (as we can see in Fig. 1).

In these cooler stars, starting at $\approx$ late $F$ types, significant excitation of hydrogen occurs only deep in the photosphere. The formation of the hydrogen lines is therefore dependent on the model in the deep layers, and this in turn hinges on what convection is doing and how it is modeled (ref. Barklem et al. 2002, Smalley \& Kupka, this conference). It will be interesting to see if multistream models or, better, hydrodynamic models yield significant improvements in our understanding of hydrogen-line wings.

I can't resist also showing a recent analysis by Bergeron \& Liebert (2002) of a composite white dwarf. Figure 2 shows their detective work in which the hydrogen lines give away the presence of the two stars.

Another powerful thermometer is the ratio of the central depths of spectral lines, typically vanadium with high temperature sensitivity to iron with lower sensitivity (e.g., Gray 1994, Strassmeier \& Schordan 2000, Gray \& Brown 2001). Figure 2 shows an example of the line behavior. As applied so far, this is not an absolute determination of temperature but rather a sophisticated interpolation 


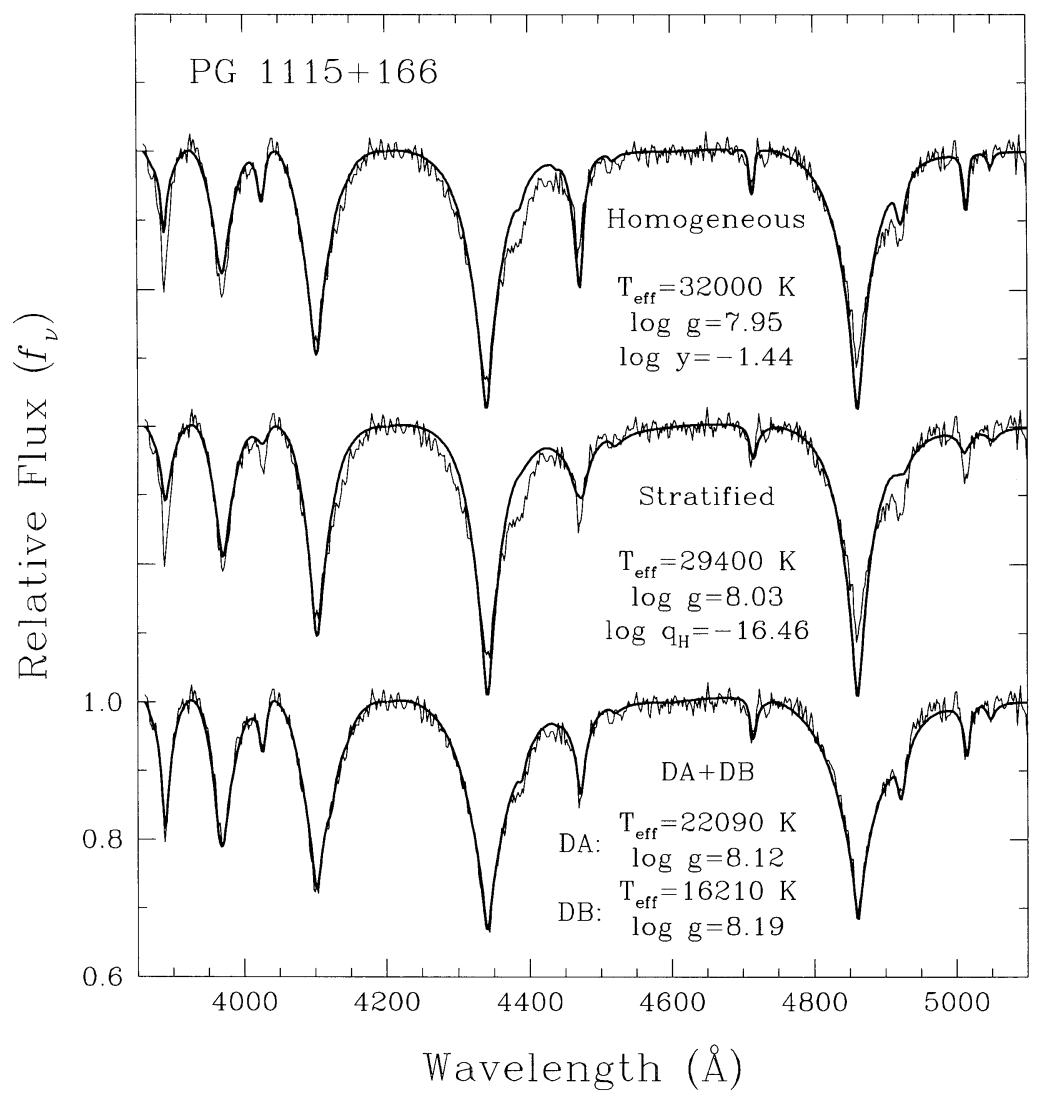

Figure 2. The Balmer lines are most successfully modeled as a composite (bottom) with the temperature and gravity parameters shown (from Bergeron \& Liebert 2002). 
device. Precision of a few degree is obtained, making this tool particularly useful for monitoring temperature variations. In principle, absolute calibration can be accomplished just as it was with the hydrogen lines discussed above, but with the difference that typical metal lines are not sensitive to the deepest photospheric layers.

\section{Irradiance Variations}

Variation of the power received on earth from the sun (the irradiance) is of obvious importance in the context of global warming, and an explanation of the observed variations offers a considerable challenge. The spectral variation that accompany the solar irradiance variations is also of interest since the response on earth depends on the spectral region (e.g., Soon et al. 2000). It seems likely that the variation in the sun's power output arises from a modulation of the power passing through the surface rather than any variation in power generated or radius change. Further, the important role played by magnetic fields is obvious from the empirical perspective. Consequently, the atmosphere of a star is a central factor in irradiance variations.

Foukal \& Lean (1988, 1990) and Lean (2000) suggested that the fraction of the surface covered by plage (magnetic region) and sunspots to be the determining factors. From the model atmosphere point of view, this is similar to the old 'two-stream,' then multi-stream, models introduced in the mid-1900s in the context of granulation. Solanki \& Unruh (1998), Unruh et al. (2000), and Fligge et al. (2000) used this same kind of model with some success; Fig. 4 shows the type of temperature distributions associated with the components they use. This model was adjusted to reproduce the total power variation and the gross features of the wavelength variations between the maximum and minimum of the solar cycle. More recently Fontenla et al. (1999) and Fox et al. (2002) expanded the concept to a seven (!) stream model. Their idea is to exploit the redundancy of information within the solar spectrum so that only a subset of measurements will allow reconstruction of the whole solar spectrum with sufficient accuracy for terrestrial climate purposes.

A simpler version of the empirical approach uses line-depth ratios in an attempt to detect and measure the variation of the temperature of the sun (Gray \& Livingston 1997). Using a run of stars covering the solar temperature portion of the main sequence, the observed solar variations were translated into temperature changes. Although the results are interesting in that the variation in line-depth ratios (C I $\lambda 5380 \AA$ to Fe I $\lambda 5379 \AA$ and Ti II $\lambda 5381 \AA$ ) gave essentially the same temperature variation one deduces from the irradiance variations (about $1.5 \mathrm{~K}$ ), the calibration can be called into question because the stars on which it is based naturally have a correlation of surface gravity with effective temperature. Caccin et al. (2002) have recently taken up this point, although their initial models, designed to handle the gravity effect on granulation, showed a correction of the wrong sign, their result did indicate that surface gravity differences may well have to be taken into account. Further, the observed wavelength dependence of the solar variation is not reproduced by a simple scaling of the model's temperature structure (Solanki \& Unruh 1998, Unruh et al. 2000); but of course this does not imply a constant effective temperature for the real sun. 

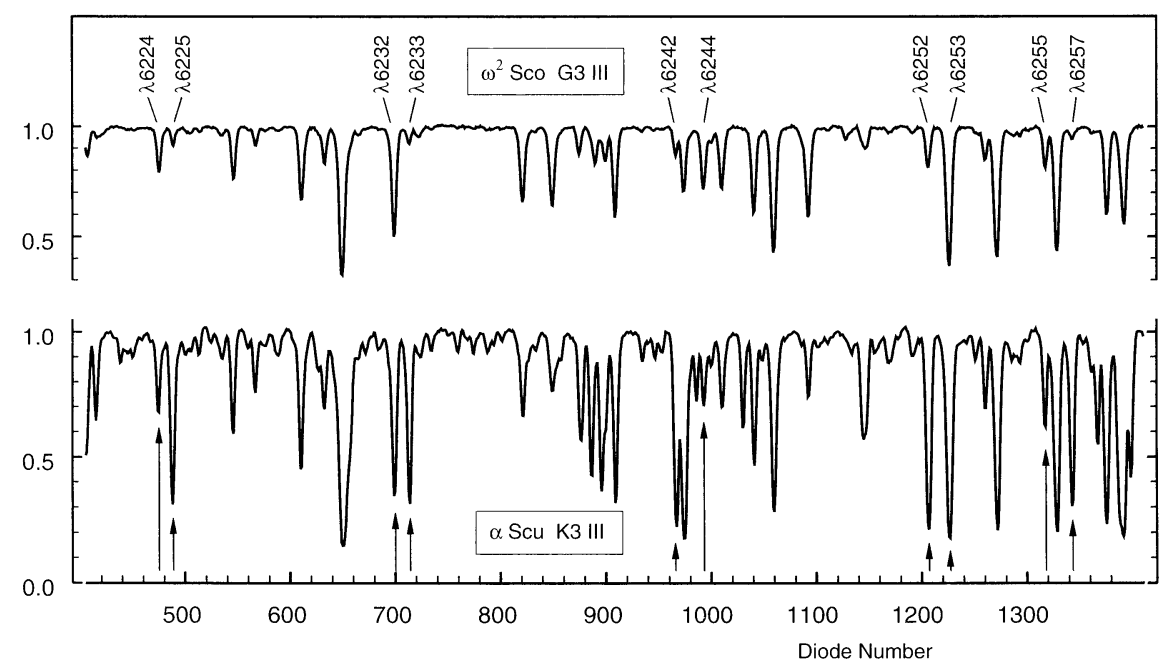

Figure 3. The central depths of close pairs of lines are used to fix temperature (from Gray \& Brown 2001).

The connection between solar activity and climate may not be simply the irradiance. Solanki et al. (2000) model a coupling through the sun's open field lines, the interplanetary field, and cosmic rays. The latter two steps are discussed in more detail by Soon et al. (2000a, 2000b), and the references therein.

\section{Stellar Rotation from Line Profiles}

Rotational broadening of spectral lines has been modeled with various degrees of complexity. In many cases, a simple rigid-rotator model matches the observations within the errors of measurement. One can usually neglect the variation in the local $I_{\nu}$ line profile across the disk, especially when $v \sin i$ is at least a few times larger than the characteristic photospheric velocity dispersion. Observational differences in the 2nd and 3rd order sidelobes from one line to the next in the same star are often fairly large, as illustrated in Fig. 5. In this example, the continuum signal-to-noise ratio is $\approx 700$. The differences arise from non-obvious line blends, errors in setting the continuum, and random noise. Similar differences were found by Dravins (1990) in his analysis of Sirius (A1 $\mathrm{V}$ ), where signal-to-noise ratios of 1000 were reached and problems with line blending are less than for cooler stars. Even so, results from the four spectral lines he measured ranged from $v \sin i=14.8$ to $15.8 \mathrm{~km} / \mathrm{s}$. Obviously some care must be used to avoid over-interpretation when more than a rotation rate is to be extracted from the data.

Occasionally, one is faced with line profiles like those in Vega (Fig. 6); they truly vary in shape from one line to the next! These have been interpreted by Gulliver et al. (1994) as a gravity effect, i.e., the star is really a rapid rotator $\left(v_{\mathrm{eq}}=245 \mathrm{~km} / \mathrm{s}\right)$ seen nearly pole-on $(v \sin i=22 \mathrm{~km} / \mathrm{s})$. That means the 


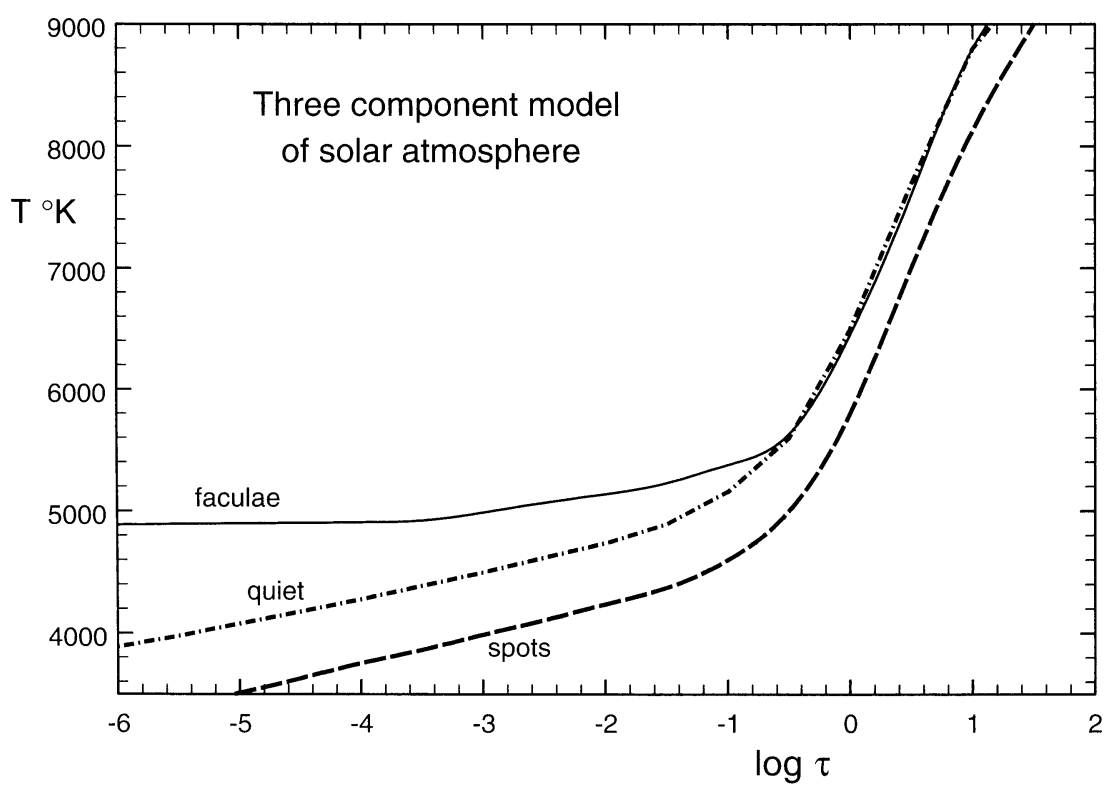

Figure 4. Temperature distributions for the three-streams used to model the variation in the solar irradiance (from Unruh et al. 2000).

limb nearly corresponds to the equator, where the rapid rotation has reduced the effective gravity enough to enhance the ionization levels of some species. Now the variation of the local specific-intensity profile has to be included in the model and numerical disk integration is mandatory. Undoubtedly a careful in-depth study of the Vega spectrum will yield interesting information about its photosphere, indeed see the paper by Bikmaev et al. at this meeting.

The spectroscopic signature of differential rotation was searched for in several stars, but not found (Huang 1961, Gray 1977, 1982b, Bruning 1981). The signature is most easily displayed in the Fourier transforms of the line profiles, where the first sidelobe shrinks with greater equatorial differential velocity. The amplitude of the second sidelobe hardly changes, and this is a crucial point that allows one to avoid ambiguity with reduced amplitudes of all sidelobes caused by photospheric velocity fields (which I will call macroturbulence). Recently Reiners et al. (2001) found evidence in two lines of $\psi$ Cap (HR 7936, HD 197692, F5 $\mathrm{V})$ for a nearly solar rate of differential rotation.

The profiles of differentially rotating stars actually depend on several variables: the equatorial velocity, the variation of rotation with latitude, the limb darkening, the aspect of view, and the size and nature of the macroturbulence. Over limited ranges in parameter space, changes in some of these variables can mimic changes in other variables, leading to ambiguities. Here let me comment on one particular point that is somewhat more elusive than the others, namely, the interaction of the Doppler shifts of macroturbulence with those of rotation. 

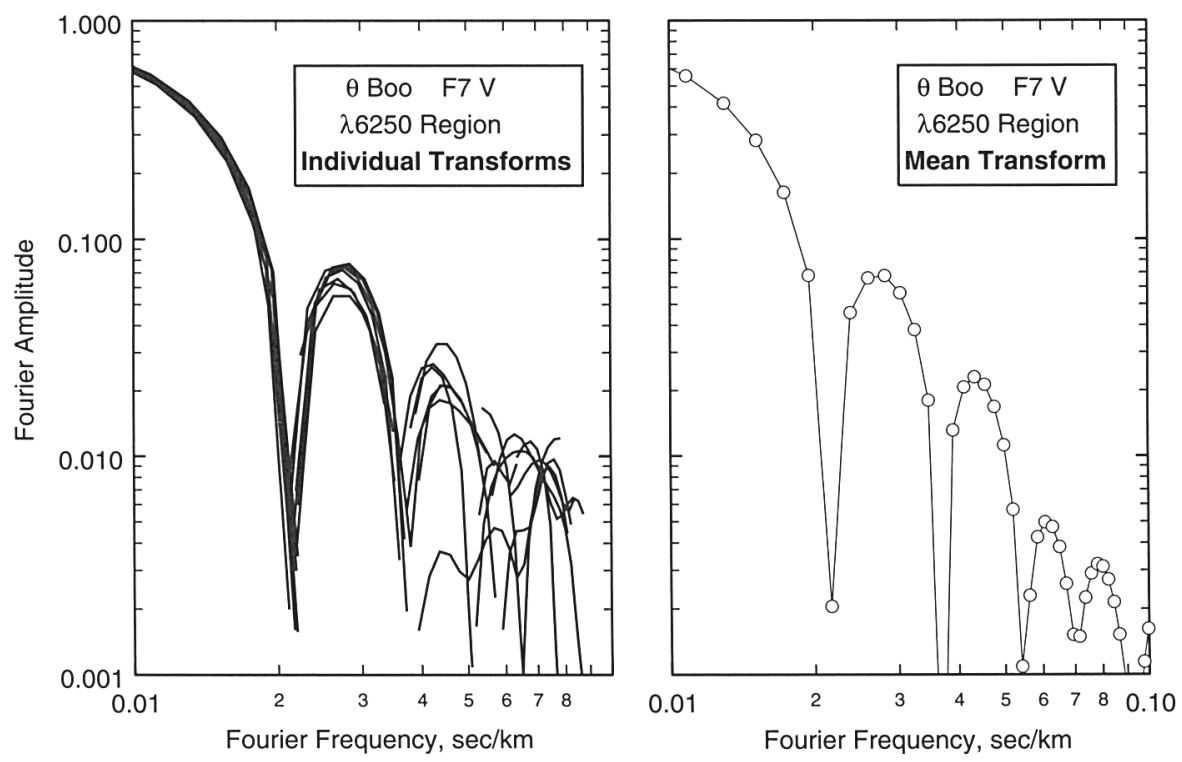

Figure 5. Fourier transforms of several spectral lines in the $\lambda 6250 \AA$ region of the spectrum of $\Theta$ Boo are shown on the left. Even with a signal-to-noise ratio $\approx 700$, there are noticeable differences in the sidelobe structure from one line to the next. On the right, the mean transform shows relatively well defined sidelobes.

If stars had isotropic macroturbulence, then every point on the disk would show the same Doppler broadening from macroturbulence. This implies that one could convolve the distribution of macroturbulent velocities with the rotation profile, i.e., the macroturbulence distribution would then be 'factorable,' and in the Fourier domain, the macroturbulence would act as a simple multiplicative filter (e.g., Gray 1988). But stars are not so simple nor accommodating. We have reasonable evidence that non-isotropic velocities are the norm. Therefore, the simple convolution is not a proper way to include macroturbulence in a model. Instead, the Doppler shifts of macroturbulence must be added to those of rotation point-by-point across the disk of the star, followed by a disk integration. It is fairly common for investigators to ignore this complication and thereby compromise the integrity of their investigation.

\section{Velocity Fields in Stellar Photospheres}

The discovery of photospheric velocity fields has a long history going back three quarters of a century (ref. Gray 1978). Stellar spectroscopists new that real spectral lines were broader than expected, and Doppler shifts from non-thermal velocities seemed the natural explanation. Early researchers introduced microturbulence as a mechanism to explain the delay of the onset of saturation in curves 


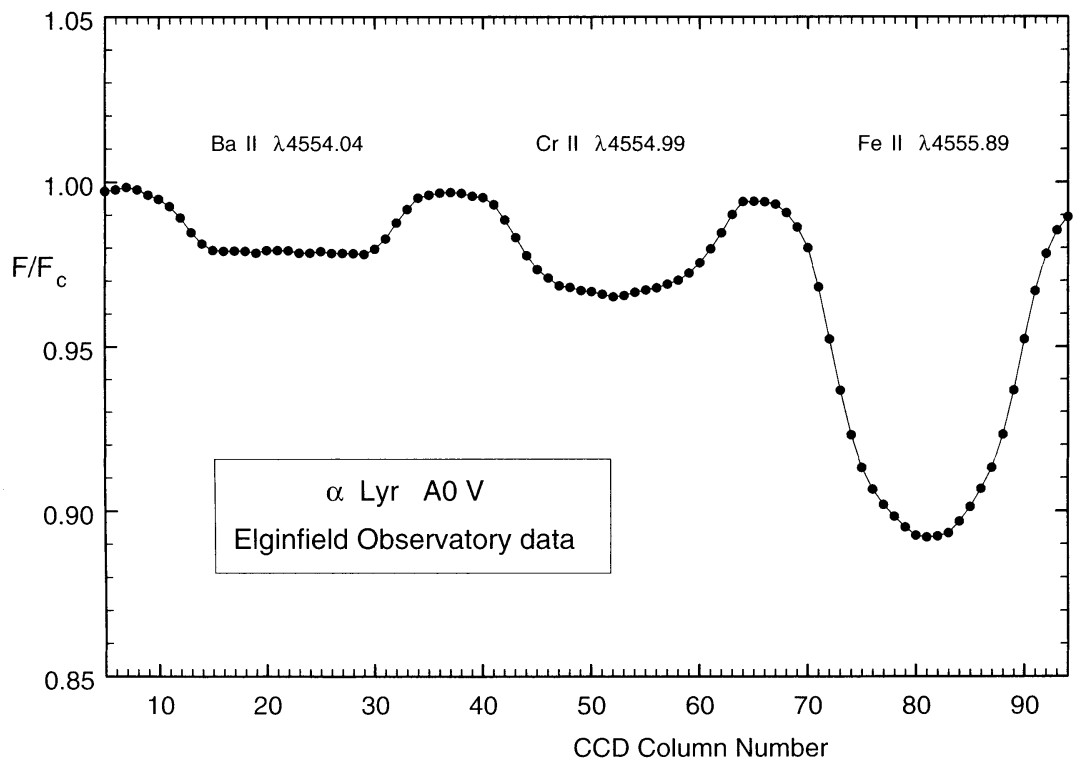

Figure 6. A sample of the profile types exhibited by Vega (HR 7001, $\mathrm{A} 0 \mathrm{~V}, v \sin i \approx 22 \mathrm{~km} / \mathrm{s}$ ). Flat bottomed profiles like the one for $\lambda 4554 \AA$ are common in Vega's spectrum; this is not a line blend.

of growth. The convolution of a Doppler-shift distribution from a thermal-like velocity field with that of the true thermal velocities made for an easy computation and has been used universally since its introduction. In 1972, Worrall \& Wilson challenged our understanding of the formation of spectral lines, saying that microturbulence was an artificial 'fudge factor' without a physical basis (see also Gray 1973, Gray \& Evans 1973). The hydrodynamical computations of recent years (e.g., Dravins \& Nordlund 1990, Stein \& Nordlund 1998, Asplund et al. 2000, and numerous others) have shown that velocity fields is indeed likely to be the correct explanation, so microturbulence may have been a 'fudge factor,' but it was not an artificial one. At the same time, the computations indicate that gradients in velocity on small optical depth scales is what we are dealing with rather than real turbulence. This also is not surprising since most astrophysicists admitted long ago that stellar turbulence was not likely to be aerodynamic turbulence.

Once higher resolution spectrographs came into the picture, macroturbulence had to be introduced as a mechanism to explain the extra broadening of lines that did not introduce any increase in equivalent widths (e.g., Huang \& Struve 1953). The introduction of the radial-tangential form of macroturbulence, along with the application of Fourier transforms to line-profile analysis, allowed the Doppler broadening of rotation to be separated from the Doppler broadening of photospheric velocity fields for stars on the cool half of the HR diagram (see Gray 1988 and references therein). The behavior of rotation across the HR 


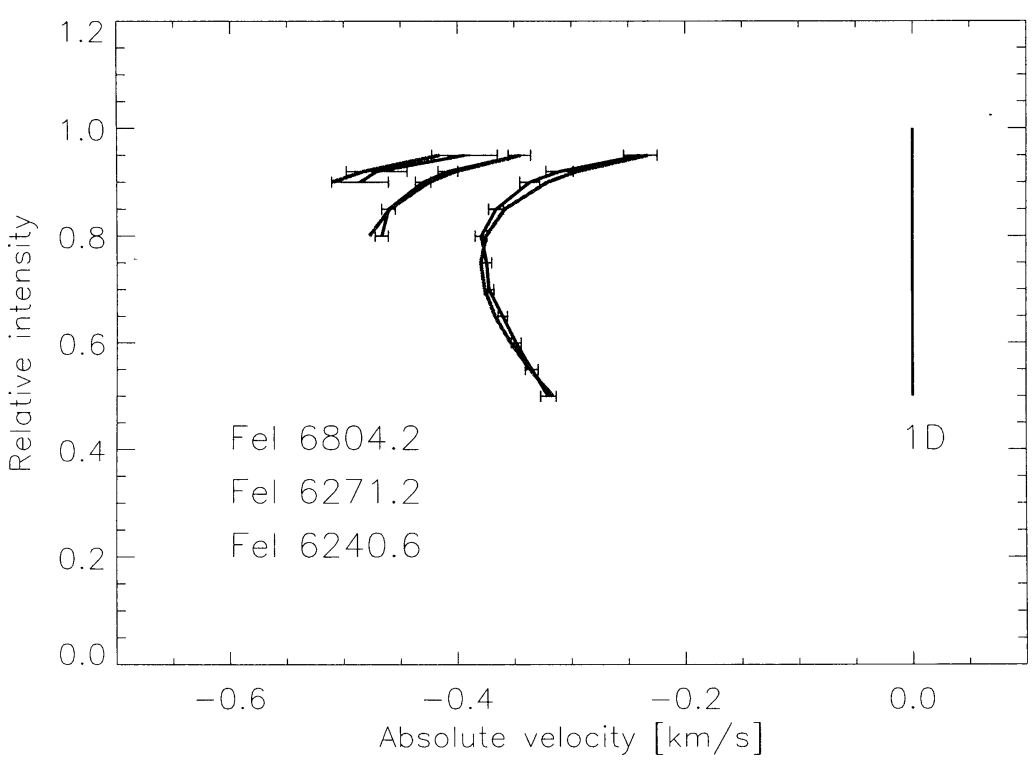

Figure 7. Observed line bisectors (with error bars) in the solar spectrum are compared to those from models. Both the absolute shift and the shape are significant here (from Asplund et al. 2000). The three lines are Fe I $\lambda 6804.2 \AA, \lambda 6271.2 \AA, \& \lambda 6240.6 \AA$.

diagram is an interesting story in its own right (ref. Gray 1991). The dispersion of photospheric velocity fields were shown to increase with effective temperature and with luminosity, ranging from $\zeta_{\mathrm{RT}} \approx 1 \mathrm{~km} / \mathrm{s}$ up to $\approx 15 \mathrm{~km} / \mathrm{s}$.

The global signature of granulation, in the form of line asymmetries, was naturally first seen in the solar spectrum (Voigt 1956, Schroeter 1957, Dravins et al. 1981, and others), but in due course also in stellar spectra (Gray 1980, 1981b, 1982a, Dravins 1987, Dravins \& Nordlund 1990). It did not take long to discover that the vigor of granulation, as expressed in the velocity span of the line bisector, varied systematically across the HR diagram, and the variation of the bisectors mimicked the behavior of the macroturbulence dispersion (Gray 1982c). In addition, the qualitative nature of the line bisectors were found to change through the middle of the HR diagram along a line dubbed the Granulation Boundary, right-leaning or C-shaped solar-type bisectors on the cool side and left-leaning or reversed-C-shaped non-solar-type on the hot side (Gray \& Toner 1986, Gray \& Nagel 1988, Gray 1989). A proper modeling of this transition across the Granulation Boundary remains to be done.

Hydrodynamical models have continued to be developed, and the solar case is rather impressively handled. The granulation morphology and statistics have been mimicked (Stein \& Nordlund 1998). The broadening, asymmetry, and shifts are well modeled, as illustrated in Fig. 7 (Asplund et al. 2000). There remain some systematic errors is bisector shapes and displacements, as discussed in Asplund et al., but it seems likely that the basic physics in now in place. 


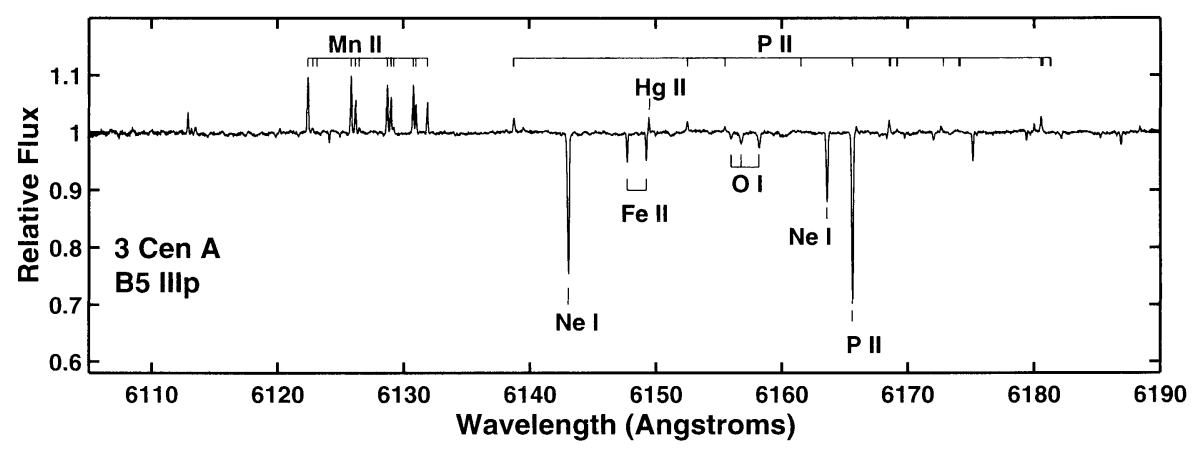

Figure 8. Non-chromospheric emission lines in the spectrum of 3 Cen A are explained with the aid of a depth-stratified Mn abundance (from Sigut et al. 2000).

The non-thermal velocities of Procyon (F5 IV-V) have been observed and modeled (Gray 1981a, 1981b, 1989, 1992, Allende Prieto et al. 2002). They are substantially larger than in the solar atmosphere with bisectors showing both velocity spans and displacements several times larger. Although the zero radial velocity has to be treated as an adjustable constant, the differential shifts in bisectors are adequately modeled in the Allende Prieto investigation, as are the bisector shapes. (Their attempt to discredit one- dimensional analyses is seriously flawed by their use of isotropic Gaussian macroturbulence instead of radial-tangential macroturbulence. In fact, the line broadening is well matched with the 1-D analysis using radial-tangential macroturbulence, but of course one needs at least a two-stream model to fabricate asymmetries.)

Procyon is moderately close to the sun in the HR diagram; the only investigations well away from the solar position of which I am aware are those of Freytag $(1999,2002)$ who is modeling the red supergiant Betelgeuse (HR 2061, M1-2, Ia-Ib). These calculations are interesting because they show as few as three or four granules on the visible disk, in general accord with Schwarschild's prediction (1975). But I have argued from the spectroscopic observations that the number of granules must be in the hundreds because the line profiles show only small variations in the Doppler-shift distribution and in addition they are always smooth and without structure (Gray 2001, 2002). This 'discussion' continues.

Some progress is being made to extend hydrodynamical computations to magnetic structures on the solar surface (e.g., Berger et al. 1998, van Ballegooijen et al. 1998.).

So, on the positive side, gains have been made by introducing basic physics of fluid dynamics and reasonable numerical integrations, calculated absolute shifts and profile shapes are close to matching the observations, and we have a better insight into the physical structure of granulation. On the negative side, the calculations are complex and not accessible to most analysts. Further, most of the HR diagram is quietly waiting to have this illumination shone on it. 


\section{Other Items}

Peculiar A stars, where we find the situation of strong but static magnetic fields, altered only by aspect of view delivered by the star's rotation. Great variety in spectral characteristics is introduced by axial orientation, nonalignment of magnetic and rotation axes, chemical migration into surface patches or stratified layers, and oscillations (e.g., Stepien \& Landstreet 2002, Kurtz et al. 2002, Girish et al. 2001, Turcotte et al. 2000).

Or consider the Mn II photospheric emission lines of 3 Cen A in Fig. 8. The explanation proposed is interlocked non-LTE effects coupled with a stratified photosphere in which the Mn abundance is some two-three orders larger in higher layers than in deeper ones (Sigut 2001). Among other things, this give support to the diffusion models of Ap stars (Michaud et al. 1974).

Observed line bisectors (with error bars) in the solar spectrum are compared to those from models. Both the absolute shift and the shape are significant here (from Asplund et al. 2000). The three lines are Fe I $6804.2 \AA, 6271.2 \AA$, \& $6240.6 \AA$. Be stars: Much of the focus is actually on surrounding disks and mass ejection events. Strengths of emission lines, continuum slope, and ionization discontinuities are used to constrain parameters in the models. See Hony et al. (2000) and Marlborough (2000).

On the tail end of the lower main sequence, large strides have been taken to understand the complex spectra (e.g., Allard et al. 2001, Ferguson et al. 2001.).

\section{Ambition}

What are some of the ambitions we might have for the future? Here are a few:

- Optical interferometry. Direct observations of stellar limb darkening should allow us to get an empirical handle on the spatial-mean temperature distributions of a sizeable sample of stars to compare with the predictions of model calculations. The results, for example, of Wittkowski et al. (2001) are encouraging, although these observations are still limited to $\sim 2$ resolution elements per stellar diameter. Interferometers also let us measure stellar oblateness (van Bell et al. this conference) resulting from rapid rotation and circumstellar disks. Spatially resolved surface features, such as the bright spot(s) on Betelgeuse, are of great interest.

- Hydrodynamical models: Application of these models to the 'analysis' domain can be expected to improve most of the parameters we extract from spectra. One of the current problems is the enormity of the calculations. Is there a simpler way to do this so we can simulate the hydrodynamical models on a desk-top computer? Can we extend hydrodynamical models away from the solar 'anchor' and across the HR diagram? Can we use them to understand the changes in granulation and line bisectors with spectral and luminosity class? In particular, can we understand the granulation boundary?

- Non-radial Oscillations: Ap stars have given us a start on modeling nonradial oscillations in stars. Can we extend this to the cool half of the HR 
diagram, especially to the evolved stars, where help is needed to sort out different mass stars with similar luminosities?

- Differential Rotation: We may be able to observe and model differential rotation for many stars. Will spectroscopic and Doppler-imaging give compatible results? Can we couple this information with the stellar activity cycles and thereby help dynamo theory along?

- Magnetic Cycles: Further observations of atmospheric changes during magnetic activity cycles should help us understand magnetic cycles, the dynamo, and possibly century-to-millennia long variations. This later understanding would be very useful in the context of global warming.

Clearly I have touched on only a very few of the many topics relevant to this meeting. Please do not feel neglected if your research has not been mentioned here; my choice of topics was rather arbitrary. Still, these topics illustrate how comparing models with observations meets with some success, some failure, significant ambiguity, but girds us up for an ambitious future. But of course, judging which endeavors fall in which categories, I leave up to you!

\section{Discussion}

GUSTAFSSON: Although Freytag's Betelgeuse simulations are as yet experimental, I suggest we should not rule them out until line profiles and line-profile navigations have been calculated for them. Note that the 'granulae' look different from those observed on the Sun, and that interesting fine structure shows up as Freytag and collaborators proceed to higher spatial resolution.

GRAY: Of course. This is a part of the on-going "discussion". There may still be some way to hide the extremely lumpy structure from the spectroscopy.

LINSKY: What are the prospects for measuring mass loss rates from the analysis of optical line profiles of solar-type dwarfs and giants?

GRAY: You know more about this than I do, Jeff. Likely, there are signatures in resonance lines or other transitions that may be set up in the escaping material.

\section{References}

Allard, F., Hauschildt, P.H., Alexander, D.R., Tamanai, A., \& Schweitzer, A. 2001, ApJ, 556, 357

Allende Prieto, C. Asplund, M., Garcia Lopez, R.J., \& Lambert, D.L. 2002, ApJ, 567, 544

Asplund, M., Nordlund, A., Trampedach, R., Allende Prieto, C., Stein, R. F. 2000, A\&A, 359, 729

Barklem, P.S., Piskunov, N. \& O'Mara, B.J. 2000, A\&A, 355, L5

Barklem, P.S., Stempels, H.C., Allende Prieto, C., Kochukhov, O.P., Piskunov, N., \& O'Mara, B.J. 2002, A\&A, 385, 951

Berger, T.E., Loefdahl, M.G., Shine, R.S., \& Title, A.M. 1998, ApJ, 495, 973 
Bergeron, P. \& Liebert, J. 2002, ApJ, 566, 1091

Bruning, D.H. 1981, ApJ, 248, 274

Caccin, B., Penza, V. \& Gomez, M.T. 2002, A\&A, 386, 286

Dravins, D. 1987, A\&A, 172, 211

Dravins, D. \& Nordlund, A. 1990, A\&A, 228, 184

Dravins, D. \& Nordlund, A. 1990, A\&A, 228, 203

Ferguson, J.W., Alexander, D.R., Allard, F., \& Hauschildt, P.H. 2001, ApJ, 557, 798

Fligge, M., Solanki, S. K., \& Unruh, Y. C. 2000, A\&A, 353, 380

Fontenla, J., White, O.R., Fox, P.A., Avrett, E.H., \& Kurucz, R.L. 1999, ApJ, 518,480

Foukal, P. \& Lean, J. 1988, ApJ, 328, 347

Foukal, P. \& Lean, J. 1990, Science 247, 505

Freytag, B. 1999, 11th Cambridge Workshop on Cool Stars, Stellar Systems, and the Sun, ASP Conf. Ser., R.J. Garcia Lopez, R. Rebolo, \& M.R. Zapatero Osorio, eds., p.785

Freytag, B. 2002, Cool Stars, Stellar Systems, and the Sun, 12, in press.

Gardiner, R.B., Kupka, F., \& Smalley, B. 1999, A\&A, 347, 876

Gehren, T. 1981, A\&A, 100, 97

Girish, V., Seetha, S., Martinez, P., Joshi, S., Ashoka, B.N., Kurtz, D.W., Chaubey, U.S., Gupta, S.K., \& Sagar, R. 2001, A\&A, 380, 142

Gray, D.F. 1973, ApJ, 184, 461

Gray, D.F. 1975, ApJ, 202, 148

Gray, D.F. 1978, Solar Phys., 59, 193

Gray, D.F. 1980, ApJ, 235, 508

Gray, D.F. 1981a, ApJ, 251, 152

Gray, D.F. 1981b, ApJ, 251, 583

Gray, D.F. 1982a, ApJ, 255, 200

Gray, D.F. 1982b, ApJ, 258, 201

Gray, D.F. 1982c, ApJ, 262, 682

Gray, D.F. 1976, The Observation and Analysis of Stellar Photospheres, (WileyInterscience: New York), 1st ed., Chap. 18

Gray, D.F. 1988, Lectures on Spectral-Line Analysis: F, G, and K Stars, (The Publisher: Arva, Ont.), Chap. 1

Gray, D.F. 1989, PASP, 101, 832

Gray, D.F. 1991, Angular Momentum Evolution of Young Stars, (Kluwer: The Netherlands), S. Catalano \& J.R. Stauffer, eds., p.183

Gray, D.F. 1992, The Observation and Analysis of Stellar Photospheres, (Cambridge: Cambridge), p.422

Gray, D.F. 1994, PASP, 106, 1248

Gray, D.F. 2000, ApJ, 532, 487

Gray, D.F. 2001, PASP, 113, 1378 
Gray, D.F. 2002, Cool Stars, Stellar Systems, and the Sun, 12, in press

Gray, D.F. \& Brown, K. 2001, PASP, 113, 723

Gray, D.F. \& Evans, J.C. 1973, J. Roy. Ast. Soc. Canada, 67, 241

Gray, D.F. \& Nagel, T. 1988, ApJ, 341, 421

Gulliver, A.F., Hill. G. \& Adelman, S.J. 1994, ApJ, 429, L81

Hony, S., Waters, L.B.F.M., Zaal, P.A., de Koter, A., Marlborough, J.M., Millar, C.E., Trams, N.R., Morris, P.W., \& de Graauw, Th. 2000, A\&A, 355, 187

Huang, S.-S. 1961, ApJ, 133, 130

Kutz, D.W. et al. 2002, MNRAS, 330, L57

Lean, J. 2000, Space Science Reviews, 94, 39

Marlborough, J.M. 2000, The Be Phenomenon in Early-Type Stars, IAU Coll. 175 , A.S.P. Conf. ser. 214,743

Michaud, G., Reeves, H., \& Charland, Y. 1974, A\&A, 37, 313

Reiners, A., Schmitt, J.H.M.M., \& Koerster, M. 2001, A\&A, 376, L13

Schwarzschild, M. 1975, ApJ, 195, 137

Sigut, T.A.A. 2001, A\&A, 377, L27

Sigut, T.A.A., Landstreet, J.D., \& Shorlin, S.L.S. 2000, ApJ, 530, L89

Smith, M.A., \& Gray, D.F. 1976, PASP, 88, 809

Solanki, S.K., Schössler, M., \& Fligge, M. 2000, Nature 408, 445

Solanki, S.K., \& Unruh, Y.C. 1998, A\&A, 329, 747

Soon, W. Baliunas, S., Posmentier, E.S., \& Okeke, P. 2000a, New Astronomy 4, 563

Soon, W., Posmentier E.S., \& Baliunas S. 2000b. Annales Geophysicae 18, 583

Stehle, C. 1994, A\&AS, 104, 509

Stehle, C. \& Hutcheon, R. 1999, A\&AS, 140, 93

Stein, R.F. \& Nordlund, A. 1998, ApJ, 499, 914

Stepien, K. \& Lanstreet, J.D. 2002, A\&A, 384, 554

Strassmeier, K.G. \& Schordan, P. 2000, Ast. Nachr. 321, 277

Turcotte, S., Richer, J., Michaud, G., \& Christensen-Dalsgaard, J. 2000, A\&A, 360,603

Unruh, Y.C., Solanki, S.K., \& Fligge, M. 2000, Space Sci. Rev. 94, 145

van Ballegooijen, A.A., Nisenson, P., Noyes, R.W., Loefdahl, M.G., Stein, R.F., Nordlund, A., \& Krishnakumar, V. 1998, ApJ, 509, 435

van't Veer-Menneret, C., Bentolila, C., \& Katz, D. 1998, Contr. Ast. Obs. Skalnate Pleso 27, 223

Worrall, G., \& Wilson, A.M. 1972, Nature 236, 15

Wittkowski, M., Hummel, C.A., Johnston, K.J., Mozurkewich, D., Hajian, A.R., \& White, N.M. 2001, A\&A, 377, 981 